\title{
Enhanced Resolution in Nanoscale NMR via Quantum Sensing with Pulses of Finite Duration
}

\author{
J. E. Lang, ${ }^{1}$ J. Casanova, ${ }^{2}$ Z.-Y. Wang, ${ }^{2}$ M. B. Plenio, ${ }^{2}$ and T. S. Monteiro ${ }^{1}$ \\ ${ }^{1}$ Department of Physics and Astronomy, University College London, Gower Street, \\ London WClE 6BT, United Kingdom \\ ${ }^{2}$ Institut für Theoretische Physik and IQST, Albert-Einstein-Allee 11, Universität Ulm, \\ D-89069 Ulm, Germany
}

(Received 16 December 2016; revised manuscript received 30 March 2017; published 15 May 2017)

\begin{abstract}
The nitrogen-vacancy $(\mathrm{N}-V)$ color center in diamond is an enormously important platform for the development of quantum sensors, including for single-spin and single-molecule NMR. Detection of weak single-spin signals is greatly enhanced by repeated sequences of microwave pulses; in these dynamicaldecoupling techniques, the key control parameters swept in the experiment are the time intervals, $\tau$, between pulses. Here, we show that, in fact, the pulse duration $t_{p}$ offers a powerful additional control parameter. While a non-negligible $t_{p}$ was previously considered simply a source of experimental error, we elucidate here the underlying quantum dynamics: we identify a landscape of quantum-state crossings which are usually inactive (closed) but may be controllably activated (opened) by adjusting $t_{p}$ from zero. We identify these crossings with recently observed but unexpected dips (so-called spurious dips) seen in the quantum coherence of the $\mathrm{N}-V$ spin. With this new understanding, both the position and the strength of these sharp features may be accurately controlled; they coexist with the usual broader coherence dips of short-duration microwave pulses, but their sharpness allows for higher-resolution spectroscopy with quantum diamond sensors, or their analogs.
\end{abstract}

DOI: 10.1103/PhysRevApplied.7.054009

\section{INTRODUCTION}

The nitrogen-vacancy $(\mathrm{N}-V)$ color center in diamond is a powerful nanoscale probe of its local environment [1-3]. The spin-1 electronic ground state can be initialized and measured using lasers via optically detected magnetic resonance, providing a window into its surroundings. A wide range of sensing techniques have been developed to perform the detection of individual nuclear spins [4-9], spin clusters [10-13], and even their manipulation for use as quantum registers [14-18].

For nuclear-spin detection, a sequence of microwave $\pi$ pulses repeatedly inverts the electronic state of the $\mathrm{N}-V$ center. If the pulse rate exceeds the width of the environmental spectral density, this permits effective decoupling from magnetic noise arising from the surrounding bath of nuclear spins, extending electronic coherence times by orders of magnitude $[19,20]$. However, wherever the pulse spacing $\tau$ is resonant with the target nuclear spins, the converse is true: system-environment interactions are magnified and a sharp signal is observed in $\mathrm{N}-V$ coherence measurements. The above mechanism underpins a wide range of techniques and pulse protocols termed dynamical decoupling (DD).

The effectiveness of DD has stimulated interest in alternative types of pulse sequences to obtain increased resolution of experimental signals and protect against pulse errors [21-23]. The only free parameters considered in these experiments thus far have been the pulse spacings and pulse phases. However, the application of certain pulse sequences has been shown to produce so-called spurious signals due to the finite duration of pulses [24,25]. To date, these finite pulses have been considered sources of error, as they lead to ambiguities in nuclear-spin classification, presenting a considerable problem for nanoscale NMR and magnetic resonance imaging (MRI) experiments.

The experimental analysis of DD has commonly been treated with semiclassical noise theory $[4,26]$ and geometric approaches [5,6], which are exact only in the simplest cases. Floquet analysis of the full quantum dynamics was recently introduced [27] as a natural framework of analysis. The method related an observed coherence dip to avoided crossings in the underlying quantum eigenstates: the shape and the depth of coherence dips are determined by the width of each avoided crossing.

In this work, we show that the avoided crossings which produce the usual coherence dips of quantum spin sensing are actually part of a much larger family of crossings. For a negligible pulse duration $\left(t_{p}=0\right)$, these are true crossings that therefore yield no signal. We show here that the effect of $t_{p}>0$ is to open-and thus to activate (to a varying extent)—this larger landscape of crossings. We develop an accurate quantum model which enables us to calculate reliably the strength of these additional signals. We identify these opened crossings with the "spurious" dips identified in recent experiments [24].

By understanding the underlying quantum dynamics, we are able to accurately model these newly identified signals: 
for a small $t_{p}$, anticrossings are narrow, so the experimental signals are sharply peaked. We can then propose how to exploit the pulse width $t_{p}$ as an alternative experimental parameter which offers an increase in resolution without the need for complex pulse sequences, i.e., utilizing the commonplace $X Y$ family [28] of pulse sequences. Furthermore, we show that spurious signals can be either enhanced or suppressed, i.e., controlled, allowing the unambiguous classification of nuclear spins. This work is not limited only to experiments with $\mathrm{N}-V$ centers: as the theory is general, it could be applied to other defect centers, such as those in silicon carbides.

The physical systems of interest here are modeled by temporally periodic Hamiltonians, $\hat{H}(t)=\hat{H}(t+T)$, where $T$ is the period. For example, for an XY4 NMR microwave pulse sequence, $T=4 \tau$, which is then repeated $N_{p}$ times. It can be shown that the behavior is given by an eigenvalue equation,

$$
\hat{H}_{F}\left|\Phi_{F}^{(n)}\right\rangle=\epsilon_{n}\left|\Phi_{F}^{(n)}\right\rangle
$$

quite analogous to a time-independent (but infinite) Schrödinger equation, but where, in place of a Hamiltonian, we diagonalize instead the (Hermitian) Floquet operator,

$$
\hat{H}_{F}=\left[\hat{H}(t)-i \frac{\partial}{\partial t}\right],
$$

to obtain the corresponding Floquet states $\left|\Phi_{F}^{(n)}\right\rangle$ and Floquet quasienergies $\epsilon_{n}$.

Previously [27], the simple case of nuclear-spin detection with a Carr-Purcell-Meiboom-Gill (CPMG) sequence $[29,30]$ of idealized, infinitely sharp $\delta$ pulses (where the pulse duration $t_{p}$ equals 0 ), allowed certain simplifications: it is, in fact, straightforward to obtain Floquet states and quasienergies by direct diagonalization of the one-period unitary $\hat{U}(T)$, constructed by concatenating the evolution between pulses. For general pulses, this procedure would not be adequate; e.g., for pulses of finite duration, the eigenvectors and quasienergies $\epsilon_{n} \equiv \epsilon_{n}\left(t_{p}\right)$ now depend on pulse duration; construction of the one-period evolution by concatenation becomes cumbersome. In this work, it becomes essential to obtain a full diagonalization of the Floquet operator with Eq. (2).

When solving Eq. (1), a usual procedure is to write the Hamiltonian,

$$
\hat{H}(t)=\hat{H}_{0}+\hat{H}_{p}(t),
$$

in terms of a time-independent component $\hat{H}_{0}$ and a timeperiodic potential $\hat{H}_{p}(t)=\hat{H}_{p}(t+T)$. For nanoscale NMR applications, $\hat{H}_{0}$ would represent the spin Hamiltonians, including Zeeman terms and spin-spin interactions, while
$\hat{H}_{p}(t)$ would represent the effect of the microwave pulses. Typically, the Floquet operator is diagonalized in the basis of $\hat{H}_{0}$. While this representation allows accurate numerical solutions, it offers comparatively little physical insight.

However, the key part of our approach is to show that we can consider instead an equivalent rotating-frame Hamiltonian,

$$
\hat{H}^{\prime}(t)=\hat{H}_{\mathrm{av}}+\hat{V}_{t_{p}}(t),
$$

where $\hat{H}_{\mathrm{av}}$ is a time-independent, average Hamiltonian, while $\hat{V}_{t_{p}}$ is an effective time-periodic potential, dependent on pulse duration, that couples the average Hamiltonian eigenstates. Section II details the transformation to this frame.

In Fig. 1, we illustrate the Floquet spectrum and the associated coherence behavior. The average spin Hamiltonian in its eigenbasis is $\hat{H}_{\mathrm{av}}=\sum_{\alpha}|\alpha\rangle\langle\alpha| \omega_{\mathrm{av}}^{\alpha}$.

(i) For the case $\hat{V}_{t_{p}}=0$, the unperturbed Floquet spectrum is shown in Fig. 1(b) and its eigenvalues are given by $\omega_{\mathrm{av}}^{\alpha}+l \omega$, where $\alpha=1, \ldots, D$, with $D$ representing the dimension of the spin system. The additional index $l=0, \pm 1, \pm 2, \ldots$ is a consequence of the invariance of Floquet states to translations in $\epsilon_{n}$ by integer multiples of $\omega=2 \pi / T$. The Floquet theorem is the temporal analog of the Bloch theorem and, analogously to the band structure seen in lattice dynamics in condensed-matter systems, the Floquet eigenspectrum has additional level structures, corresponding to quasienergy shifts of single quanta in $\omega$. Hence, the unperturbed spectrum has degeneracies wherever a pair of eigenvalues $\omega_{\alpha}, \omega_{\alpha^{\prime}}$ differ by an integer multiple of $\omega$ (see below). However, in this case, degeneracies yield true crossings and no coherence dip is seen.

(ii) For the case $\hat{V}_{\left(t_{p}=0\right)}$, when we apply $\pi$ pulses which approximate ideal $\delta$ spikes, some crossings become anticrossings and the usual structure of coherence dips appears. The width of the crossing (and hence the dip shape and visibility) depends crucially on the matrix elements of the Floquet operator. In the dressed-state basis where $|l\rangle=e^{i l \omega t}$,

$$
\begin{aligned}
\left\langle m_{s} \alpha l\left|\hat{H}_{F}\right| m_{s}^{\prime} \alpha^{\prime} l^{\prime}\right\rangle= & \left(\omega_{\mathrm{av}}^{\alpha}+l \omega\right) \delta_{\alpha \alpha^{\prime}} \delta_{l l^{\prime}} \delta_{m_{s} m_{s}^{\prime}} \\
& +\left\langle m_{s} \alpha l\left|\hat{V}_{t_{p}}\right| m_{s}^{\prime} \alpha^{\prime} l^{\prime}\right\rangle .
\end{aligned}
$$

Whether or not a dip is seen can be understood, to first order, if we consider the diagonal contributions to be dominant, so the levels may still be labeled by the unperturbed basis $m_{s} \alpha l$. If the off-diagonal matrix element of $\hat{V}_{t_{p}}$ is nonzero, then the degeneracy between the corresponding levels will be lifted, creating an avoided crossing which causes a coherence dip. For $t_{p}=0$ only, 
(a) Multispin detection
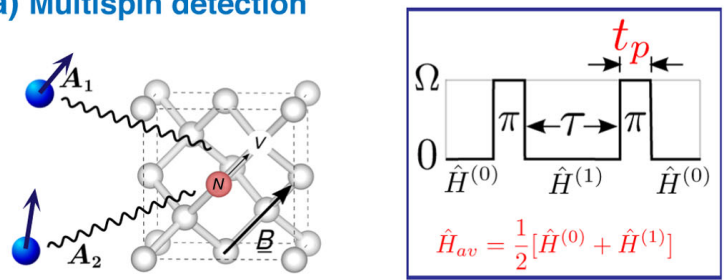

(c) Floquet spectrum for $\Delta$ pulses $t_{p}=0$

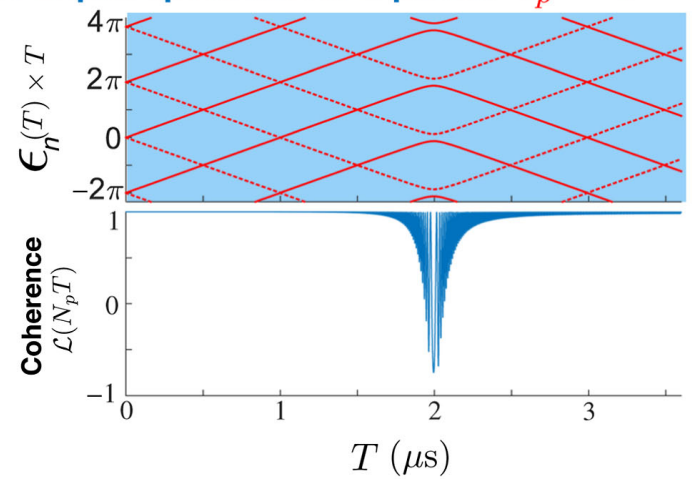

(b) Floquet spectrum of average Hamiltonian $\hat{H}_{\mathrm{av}}$

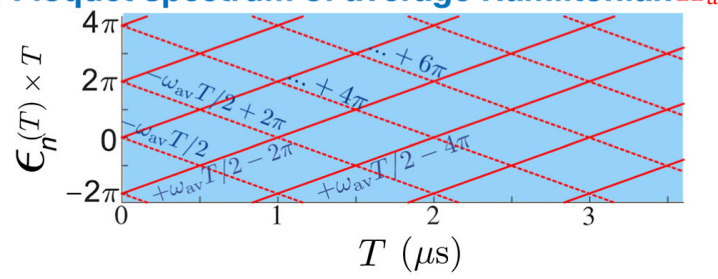

(d) Floquet spectrum for finite pulses $t_{p} \neq 0$

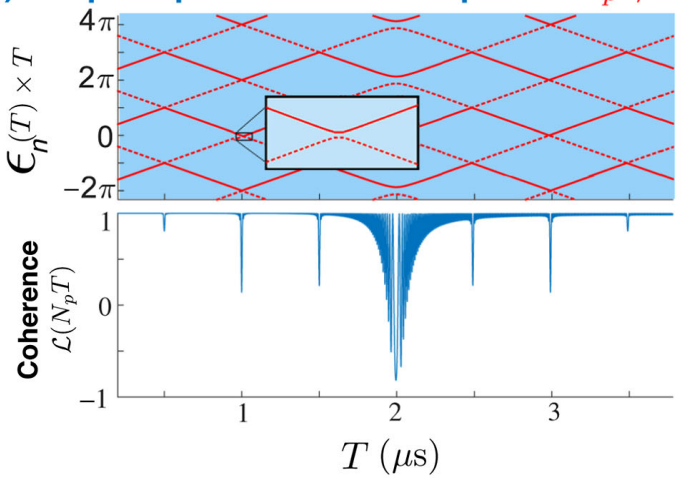

FIG. 1. (a) DD pulse sequences are commonly applied in sensing protocols using $\mathrm{N}-V$ centers. A series of microwave pulses repeatedly flip the electronic spins between an $\left|m_{s}= \pm 1\right\rangle$ and an $\left|m_{s}=0\right\rangle$ state. A frequent convenient approximation is to assume that the dynamics of an environmental spin is given by a period-averaged Hamiltonian $\hat{H}_{\mathrm{av}}$ (see Sec. II for details), with dips in coherence occurring at the pulse spacing $\tau_{\text {dip }} \sim \pi / \omega_{\mathrm{av}}$. We demonstrate in Sec. IV that spurious dips can be used to increase resolution for the detection of multiple isolated spins. [ $\hat{H}^{\left(m_{s}=0,1\right)}$ is the (N-V state-dependent) nuclear Hamiltonian.] (b) The Floquet eigenspectrum for $\hat{H}_{\text {av }}$ is given by $\omega_{\text {av }}$, but it shows the characteristic Floquet structure of dressed states shifted by integer multiples of $\omega=2 \pi / T$ (sometimes termed "multiphoton states"). In this case, level degeneracies correspond to true crossings. (c) The effect of the DD pulses, for the ideal $t_{p}=0$ case, is to turn some crossings into avoided crossings. A coherence dip, of a strength determined by the width of the crossing, can then be seen. (d) For the general $t_{p} \neq 0$ case, all of the remaining crossings can potentially become avoided crossings: typically narrow, they yield sharp weaker dips. Our main finding is to show that we can determine these dips analytically; a key result of this work is Eq. (15), where we give a closed-form expression for the shape and the strength of these so-called spurious coherence dips and argue that they are, in fact, useful for sensing. For the numerical simulations here, $\omega_{\mathrm{av}}=2 \pi \times 2 \mathrm{MHz}$ and $A_{\perp}=2 \pi \times 200 \mathrm{kHz}$. The pulse sequence is $X Y 8$, with $N_{p}=N / 8=60$ repetitions. The finite pulses have a height $\Omega=2 \pi \times 20 \mathrm{MHz}$ and a duration $t_{p}=\pi / \Omega$. $T$ is in microseconds.

$\left\langle m_{s} \alpha l\left|\hat{V}_{t_{p}}\right| m_{s}^{\prime} \alpha^{\prime} l^{\prime}\right\rangle \delta_{m_{s} m_{s}^{\prime}} \neq 0$ : for the usual dynamicaldecoupling scenario, the bath evolution in the two subspaces corresponding to the electronic states of the $\mathrm{N}-V$ sensor remains fully independent.

(iii) Finally, we consider and illustrate in Fig. 1(c) the general case of $t_{p} \neq 0$, where we apply $\pi$ pulses of finite duration. In this case, we find there are nonzero couplings for the case $m_{s} \neq m_{s}^{\prime}$, so that, potentially, all crossings here are avoided crossings. These couplings connect previously uncoupled (albeit degenerate) levels, hence turning true crossings into avoided crossings and generating new, spurious coherence dips.

The paper is constructed as follows: In Sec. II, we explain how to construct the Floquet Hamiltonian matrix and, specifically, how to calculate explicitly the matrix elements of $\hat{V}_{t_{p}}$. We exploit the fact that the dynamics at an avoided crossing reduces to an effective two-state system in order to obtain analytical forms for the coherence functions.
In Sec. III, we apply the method to the sensing of hyperfine coupled spins and show how to obtain analytically the shape and magnitude of the coherence dip for pulses of an arbitrary duration $t_{p}$ and for pulse sequences with arbitrary pulse positions. Tuning the pulse sequence can open and close avoided crossings, thus allowing new control over both expected and spurious coherence dips, and hence the effective coupling to nuclear targets. A key result of this work is an expression for spurious coherence dips given in Eq. (15) and shown, in Sec. III C, to be in excellent agreement with full numerics. In Sec. IV, we demonstrate how the small width of spurious avoided crossings equates to a significant increase in spectral resolution and, in Sec. V, we conclude.

\section{CONSTRUCTING THE FLOQUET HAMILTONIAN}

For the detection of nuclear-spin clusters via a $\mathrm{N}-\mathrm{V}$ center, it is typical to give the Hamiltonian in the form 
$\hat{H}(t)=\hat{H}_{0}+\hat{H}_{p}(t) \equiv \hat{\mathbb{}} \otimes \hat{H}_{\mathrm{av}}+\hat{\sigma}_{z} \otimes \hat{V}+\hat{H}_{p}(t)$,

which is in the form of Eq. (3). A distinction is now made between the $\mathrm{N}-V$ and nuclear-spin subspaces. Here, $\hat{\mathbb{I}}$ and $\hat{\sigma}_{z}$ are Pauli matrices in the $\mathrm{N}-V m_{s}=0,1$ subspace, $\hat{H}_{\mathrm{av}} \equiv$ $\frac{1}{2}\left[\hat{H}^{(0)}+\hat{H}^{(1)}\right]$ and $\hat{V} \equiv \frac{1}{2}\left[\hat{H}^{(1)}-\hat{H}^{(0)}\right]$ are the nuclear average and interaction Hamiltonians, respectively. Here, $\hat{H}^{\left(m_{s}=0,1\right)}=\left\langle m_{s}\left|\hat{H}_{0}\right| m_{s}\right\rangle$ is the nuclear-spin Hamiltonian conditioned on the N-V state; see Appendix A for further details.

In the frame rotating at the microwave frequency, the pulse-sequence Hamiltonian takes the form

$$
\hat{H}_{p}(t)=\Omega_{x}(t) \hat{S}_{x}+\Omega_{y}(t) \hat{S}_{y},
$$

where $\hat{S}_{x, y}$ represents the N-V spin operators and $\Omega_{x, y}(t)$ describes the amplitude of the microwave driving as a function of time.

In order to obtain the Hamiltonian in the required form of Eq. (4), we introduce a frame that rotates under $\hat{H}_{p}(t)$. That is, $\hat{H}^{\prime}(t)=\hat{U}_{p}^{\dagger}(t) \hat{H}_{0} \hat{U}_{p}(t)$, where $\hat{U}_{p}(t)=$ $\mathcal{T} \exp \left[-i \int_{0}^{t} \hat{H}_{p}\left(t^{\prime}\right) d t^{\prime}\right]$ is the time-ordered propagator.

The $\hat{H}_{\text {av }}$ component is unaffected by this transformation, but the remainder will yield the coupling matrix $\hat{V}_{t_{p}}$ of Eq. (4):

$$
\hat{V}_{t_{p}}(t)=\hat{U}_{p}^{\dagger}(t) \hat{\sigma}_{z} \hat{U}_{p}(t) \otimes \hat{V}=\sum_{i=x, y, z} f_{i}(t) \hat{\sigma}_{i} \otimes \hat{V} .
$$

Typically, microwave pulses are modeled as infinitely sharp $\delta$ pulses. This approximation is adequate whenever $t_{p}$ is much less than the nuclear signal period, so the detected spin states do not evolve appreciably over the duration of the pulse; only a modest number of pulse-sequence repetitions $N_{p}$ are applied so that cumulative errors arising from these effects remain insignificant.

In this case, the coupling matrix reduces to $\hat{V}_{t_{p}}(t)=$ $f_{z}(t) \sigma_{z} \otimes \hat{V}$, where $f_{z}(t)$ is the usual stepped modulation function [see Fig. 2(a)] defined in previous studies, and there are no $x$ or $y$ modulation functions. In this picture, the new frame instantaneously flips along the $z$ axis at each pulse. We show here that the transformation to a rotating frame is equally applicable for pulses of finite width.

As found in recent studies [24], the finite duration of pulses must be taken into account, especially where an increasing number of pulses are applied: the total phase accumulation time during pulses $N t_{p}$ can become comparable or even greater than during $\omega_{\mathrm{av}}^{-1}$ if the number of pulses, $N$, is sufficiently large. We now obtain the modulation functions for the finite-duration pulses.

\section{A. The $f_{x, y, z}(t)$ modulation functions for an arbitrary $t_{p}$}

Although Eq. (8) is generic, for convenience, we test the method on pulses modeled by top-hat functions with a
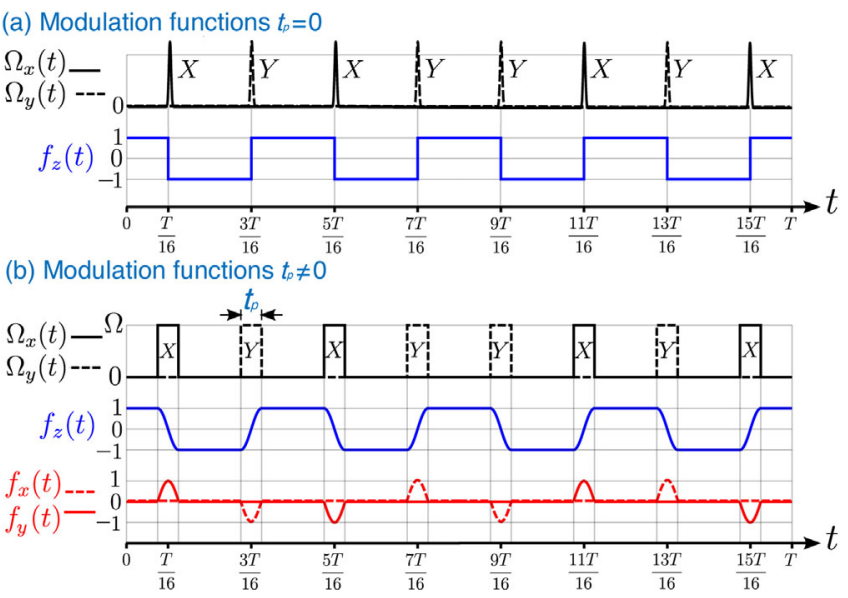

(c) Coupling-matrix elements between dressed states

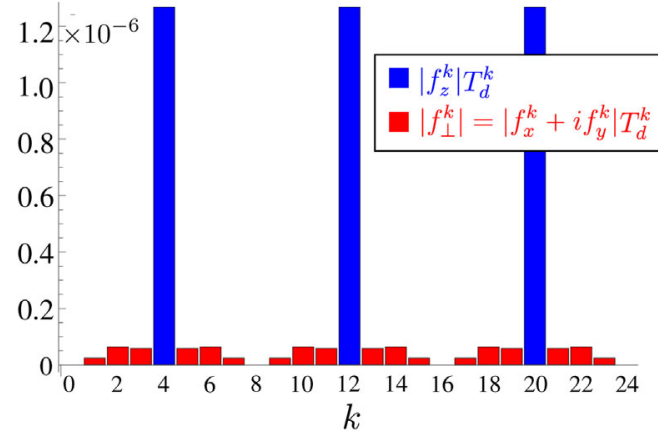

FIG. 2. Shows the modulation functions for an $X Y 8$ sequence with (a) ideal $\pi$ pulses and (b) pulses which provide a $\pi$ rotation, but which are of a finite duration $t_{p}$. For this case, there are nonzero contributions from $f_{x, y}(t)$ modulation functions which can couple the $m_{s}$ subspaces. In our quantum Floquet treatment, the modulation functions replace and generalize the role played by the filter function in classical noise treatments of dynamicaldecoupling-based sensing. (c) Matrix elements of the modulations function $\left\langle l+k\left|f_{i}\right| l\right\rangle$ in the dressed-state basis. The $f_{z}$ matrix elements (blue) for $k=4,12,20, \ldots$ are responsible for the "expected" coherence dips and are of a much larger magnitude than the $f_{x, y}$ matrix elements, which are nonzero only for $t_{p}>0$ and give rise to the so-called spurious coherence dips. CPMG control does not produce spurious signals, precisely because, in that case, the nonzero $f_{\perp}^{k}$ and $f_{z}^{k}$ 's coincide. The CPMG case is discussed in Appendix C.

given finite height $\Omega$ and width $t_{p}=\pi / \Omega$, chosen such that a full $\pi$ rotation is completed during the pulse. In this case, during the pulse, the frame does not instantaneously flip along the $z$ axis but must evolve smoothly between the initial and final positions. This transit induces a sinusoidal curve between \pm 1 during the pulse, as seen in Fig. 2(b). The unitarity of the transformation to the rotating frame then requires that the transverse modulation functions, $f_{x, y}(t)$, be nonzero [again, see Fig. 2(b)].

During the pulse, the frame rotates about some axis in the $x-y$ plane, with a phase determined by the phase of the pulse being applied and also by the phases of the pulses that 
came before it. The rotation is such that a unit vector $+\hat{\mathbf{z}}$ becomes $-\hat{\mathbf{z}}$ by the end of the pulse.

For an isolated top-hat pulse, the transformation of $\hat{\sigma}_{z}$ is simple to calculate; however, the history of previous pulses must also be taken into account. Consider an arbitrary sequence of $n$ pulses, all of height $\Omega$ and length $t_{p}=\pi / \Omega$, applied at times $\left\{t_{1}, \ldots, t_{n}\right\}$ and phases $\left\{\phi_{1}, \ldots, \phi_{n}\right\} \quad(\phi=0$ and $\phi=\pi / 2$ correspond to $X$ and $Y$ pulses, respectively). During the $m$ th pulse, i.e., for $t^{\prime}=t-t_{m} \in\left[-t_{p} / 2,+t_{p} / 2\right]$,

$$
\begin{aligned}
\hat{U}_{p}\left(t^{\prime}\right)= & \exp \left[-i \Omega \hat{S}_{\phi_{m}}\left(t^{\prime}+t p / 2\right)\right] \\
& \times\left(-i \hat{\sigma}_{\phi_{m-1}}\right) \times \cdots \times\left(-i \hat{\sigma}_{\phi_{1}}\right) .
\end{aligned}
$$

Thus, we have

$$
\begin{aligned}
\hat{U}_{p}^{\dagger}\left(t^{\prime}\right) \hat{\sigma}_{z} \hat{U}_{p}\left(t^{\prime}\right)= & (-1)^{m} \sin \Omega t^{\prime} \sigma_{z}+\cos \Omega t^{\prime} \cos \varphi_{m} \sigma_{x} \\
& +\cos \Omega t^{\prime} \sin \varphi_{m} \sigma_{y},
\end{aligned}
$$

where $\varphi_{m}=2 \sum_{k=1}^{m-1}(-1)^{k+1} \phi_{k}+(-1)^{m+1}\left(\phi_{m}+\pi / 2\right)$. The modulation functions during the $m$ th pulse are

$$
\begin{aligned}
& f_{x}\left(t^{\prime}\right)=\cos \Omega t^{\prime} \cos \varphi_{m}, \\
& f_{y}\left(t^{\prime}\right)=\cos \Omega t^{\prime} \sin \varphi_{m}, \\
& f_{z}\left(t^{\prime}\right)=(-1)^{m} \sin \Omega t^{\prime},
\end{aligned}
$$

and, between the pulses, $f_{z}(t)= \pm 1$ and $f_{x, y}(t)=0$.

In this section, we define an expanded set of modulation functions for realistic pulse sequences that generalize the stepped-modulation function used to model infinitely sharp microwave pulses. These modulation functions can be calculated for any periodic pulse sequence, and they are crucial for deriving an analytical model of the spurious signals produced by realistic pulses.

\section{B. Matrix elements of the coupling $\hat{\boldsymbol{V}}_{t_{p}}$}

Now that the modulation functions $f_{i}(t)$ have been obtained, the matrix elements of $\hat{V}_{t_{p}}$ may be straightforwardly evaluated in the $\left|m_{s} \alpha l\right\rangle$ basis:

$$
\left\langle m_{s} \alpha l\left|\hat{V}_{t_{p}}\right| m_{s}^{\prime} \alpha^{\prime} l^{\prime}\right\rangle=\sum_{i=x, y, z} f_{i}^{l-l^{\prime}}\left\langle m_{s}\left|\hat{\sigma}_{i}\right| m_{s}^{\prime}\right\rangle\left\langle\alpha|\hat{V}| \alpha^{\prime}\right\rangle .
$$

The $\left\langle m_{s}\left|\hat{\sigma}_{i}\right| m_{s}^{\prime}\right\rangle$ 's are common Pauli matrix elements, while evaluating the nuclear-interaction Hamiltonian matrix elements $\left\langle\alpha|\hat{V}| \alpha^{\prime}\right\rangle$ is a standard procedure and is typically quite straightforward: for nuclear-spin detection $\hat{V}=A_{\perp} \hat{I}_{x}$ (see Appendix B for further details).

However, the modulation-function matrix elements in the dressed-state basis are of most significance: $f_{i}^{k}=\left\langle l+k\left|f_{i}(t)\right| l\right\rangle=\frac{1}{T} \int_{0}^{T} d t^{\prime} f_{i}\left(t^{\prime}\right) \exp \left(-i k \omega t^{\prime}\right)$

are the Fourier amplitudes of the periodic modulation functions. They play a key role in our approach by selecting whether a crossing becomes an anticrossing, as well as determining its width; in effect, they replace (and, for the $f_{\perp}$ case, generalize) the role played by the filter function, a common method of analysis of spin sensing, using classical noise models.

Importantly, for robust DD sequences (see Fig. 2), $f_{z}^{k}$ and $f_{\perp}^{k}=f_{x}^{k}+i f_{y}^{k}$ are nonzero for different values of $k$. The $f_{z}^{k}$ terms create avoided crossings and coherence dips at expected pulse spacings, whereas $f_{\perp}^{k}$ creates new avoided crossings and dips at so-called spurious positions. The width of the avoided crossing is proportional to the magnitude of the relevant $f_{z}^{k}$ or $f_{\perp}^{k}$. The fact that $\left|f_{z}^{k}\right| \gg$ $\left|f_{\perp}^{k}\right|$ immediately explains why the spurious dips are much sharper and are typically seen only for large pulse numbers, which is a result of narrower avoided crossings. This understanding of spurious dip sharpness leads us to propose a sensing protocol that exploits this behavior to obtain increased spectral resolution. This method is discussed in Sec. IV. The finite-pulse effect in experiments using a CPMG sequence is discussed in Appendix C.

\section{APPLICATION: SINGLE-SPIN NMR}

\section{A. Average Hamiltonian states}

For the detection of a single spin $1 / 2$, such as an environmental nuclear spin, the average Hamiltonian $\hat{H}_{\mathrm{av}}=\omega_{\mathrm{av}} \hat{I}_{z}$ and $\hat{V}=A_{\perp} \hat{I}_{x} ;$ hence, the average Hamiltonian basis takes a very simple form. Figure 3 and Appendix A describe how the average Hamiltonian basis relates to the Zeeman basis. In the weak-coupling

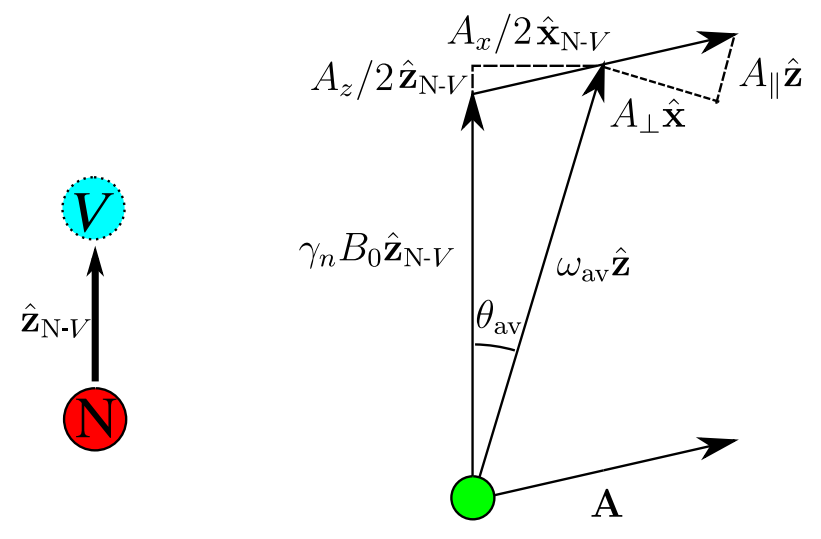

FIG. 3. The direction between the nitrogen and vacancy sites in the $\mathrm{N}-V$ center sets the axis $\hat{\mathbf{z}}_{\mathrm{N}-V}$, and the external field, $\mathbf{B}=B_{z} \hat{\mathbf{z}}_{\mathrm{N}-V}$, is applied parallel. The hyperfine field felt at the nucleus, $\mathbf{A}=\left(A_{x}, 0, A_{z}\right)$, shifts the quantization axes of the nuclear Hamiltonian. It is useful to work in the nuclear average Hamiltonian basis, and this quantization axis is shown. 
regime, $A_{\perp} \ll \omega_{\mathrm{av}}$, so the diagonal matrix elements of $\hat{H}_{F}$ are much larger than the off-diagonal couplings. The unperturbed Floquet eigenspectra is given by $\left\{ \pm \omega_{\mathrm{av}} / 2+\right.$ $l \omega \mid l=0, \pm 1, \pm 2, \ldots\}$. Degeneracies occur in the spectra when $\omega=\omega_{\mathrm{av}} / k$, i.e., $T=2 \pi k / \omega_{\mathrm{av}}$. Figure 1(b) shows the unperturbed eigenspectra in a scan of DD period, $T$.

The off-diagonal terms in the $\hat{V}_{t}$ coupling matrix are $A_{\perp} f_{z}^{k} / 2$ and $A_{\perp} f_{\perp}^{k} / 2=A_{\perp}\left(f_{x}^{k}+i f_{y}^{k}\right) / 2$, where $f_{i}^{k}$ represents the Fourier amplitudes of the modulation functions. Figure 2 shows the magnitude of the Fourier amplitudes for an $X Y 8$ pulse sequence evaluated at the degeneracies $T=2 \pi k / \omega_{\mathrm{av}}$. The coherence dips expected from DDbased sensing are caused by $f_{z}^{k}$; thus, for an $X Y 8$ sequence, dips occur at $T=2 \pi k / \omega_{\text {av }}$ for $k=4,12,20, \ldots$, as seen in
Fig. 1(c). When a finite pulse is modeled, the $f_{\perp}^{k}$ 's become nonzero, opening new avoided crossings and causing new coherence dips. The resulting perturbed eigenspectra with avoided crossings are illustrated in Fig. 1(d).

\section{B. Calculating the coherence function}

To model a spurious dip, $\hat{H}_{F}$ must be diagonalized at $T \approx 2 \pi k / \omega_{\text {av }}$, with $k$ 's such that $f_{\perp}^{k}=\left|f_{\perp}^{k}\right| \exp \left(i \phi_{\perp}^{k}\right) \neq 0$. At this value of $T$, the Floquet Hamiltonian can be treated as an infinite set of $2 \times 2$ matrices as the pairs of degenerate diagonal entries, $\omega_{\mathrm{av}} / 2+l \omega$ and $-\omega_{\mathrm{av}} / 2+(l+k) \omega$, are coupled by the $f_{\perp}^{k}$ (or $f_{\perp}^{-k}$ ) terms, whereas all other offdiagonal entries fail to couple a degeneracy and can be neglected. The procedure is shown in Fig. 4, which

(a) Floquet Hamiltonian

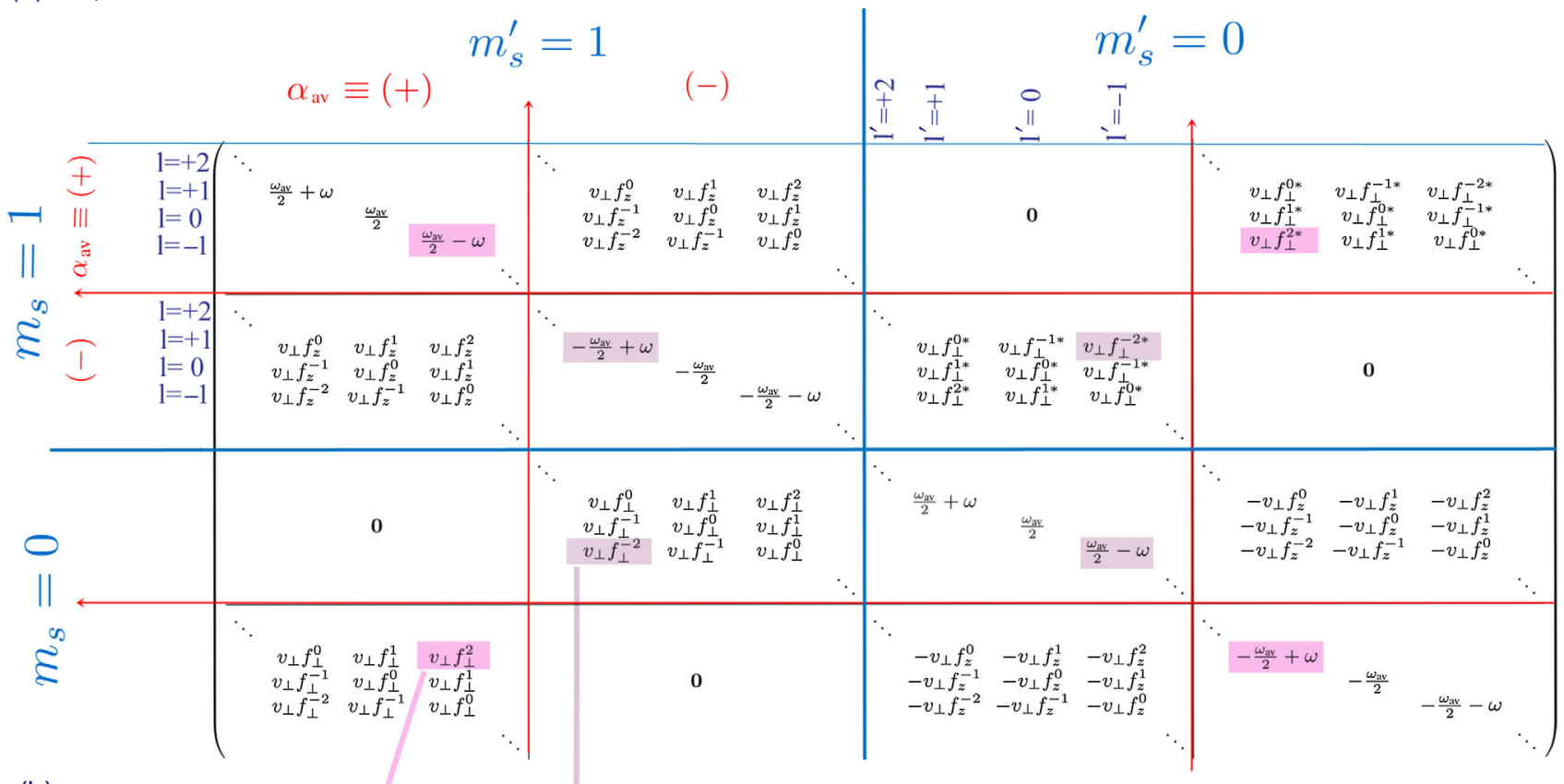

(b)

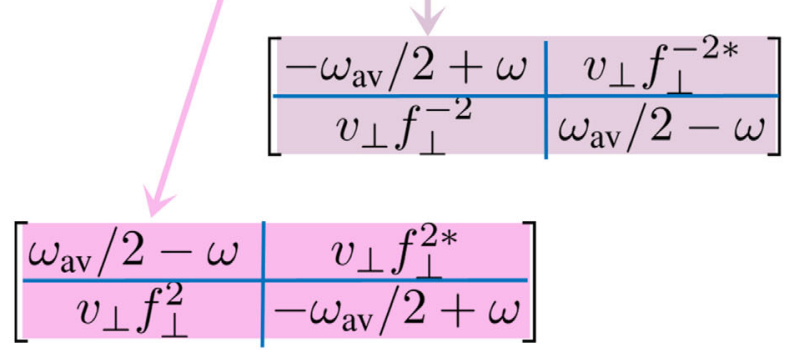

(c)

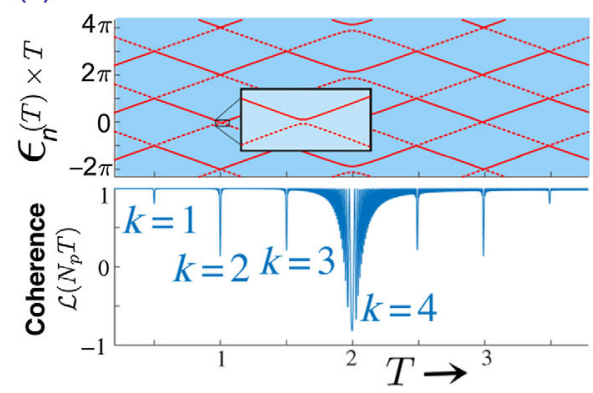

FIG. 4. Toolbox for calculating coherence function for finite pulses for detection of spin-half environmental spins. (a) Schematic representation of the Floquet Hamiltonian matrix. Each element of the original Hamiltonian becomes an infinite block containing all of the Fourier coefficients. The relative magnitude of the off-diagonal terms are determined by the hyperfine coupling strength $v_{\perp}=A_{\perp} / 2$ and the Fourier series of the periodic modulation functions, $f_{z}^{k}$ (for the $m_{s}=m_{s}^{\prime}$ case, ordinary coherence dips) and $f_{\perp}^{k}=f_{x}^{k}+i f_{y}^{k}$ (when $m_{s} \neq m_{s}^{\prime}$, spurious dips). (b) To evaluate the form of the $k=2 \mathrm{dip}$, we consider the coupling, at $T \approx T_{\mathrm{dip}}^{(k=2)}$, between the pairs of states in the avoided crossing. The resulting $2 \times 2$ matrices can be diagonalized exactly to yield the stroboscopic evolution (see Sec. III B) and an analytical expression for the shape of the coherence dip shown in (c). 
illustrates the specific case of a $k=2$ dip. The Floquet Hamiltonian is effectively decoupled into $2 \times 2$ subspaces, and they can be diagonalized analytically.

The Floquet Hamiltonian can then be written in the form $\hat{H}_{F}=\hat{D}_{F} \hat{\Lambda}_{F} \hat{D}_{F}^{-1}$, where $\hat{\Lambda}_{F}$ is the diagonal matrix of Floquet quasienergies and $\hat{D}_{F}$ contains all of the Floquet Hamiltonian eigenstates. The stroboscopic evolution operator is given by $\hat{U}\left(N_{p} T\right)=\hat{D} \exp \left(-i \hat{\Lambda} N_{p} T\right) \hat{D}^{-1}$, where $\left\langle m_{s} \alpha|\hat{D}| m_{s}^{\prime} \alpha^{\prime}\right\rangle=\sum_{l}\left\langle m_{s} \alpha l\left|\hat{D}_{F}\right| m_{s}^{\prime} \alpha^{\prime} 0\right\rangle$ and $\left\langle m_{s} \alpha|\hat{\Lambda}| m_{s} \alpha\right\rangle=$ $\left\langle m_{s} \alpha 0\left|\hat{\Lambda}_{F}\right| m_{s} \alpha 0\right\rangle$. This expression is valid for arbitrary $N_{p}$ 's (and given explicitly in Appendix B), and thus an analytic expression for the spurious coherence response of the $\mathrm{N}-V$ center can be obtained:

$$
\mathcal{L}\left(N_{p} T\right) \propto\left\langle\hat{S}_{x}\right\rangle=\operatorname{Tr}\left[\hat{S}_{x} \hat{U}\left(N_{p} T\right) \rho_{0} \hat{U}^{\dagger}\left(N_{p} T\right)\right] .
$$

Typically, the $\mathrm{N}-V$ center is initially prepared in a superposition state along the $+x$ direction and the nuclear target is assumed to be in a thermal state. The initial density matrix is $\rho_{0}=\frac{1}{4}\left(\mathbb{\square}+\sigma_{x}\right)_{\mathrm{N}-V} \otimes \mathbb{I}_{\text {target }}$.

The spurious coherence dip is given by

$$
\begin{aligned}
\mathcal{L}_{s}\left(N_{p} T\right)= & 1-2\left\{\frac{\epsilon_{s}(k ; T)^{2}-\left(\omega_{\mathrm{av}}-k \omega\right)^{2} / 4}{\epsilon_{s}(k ; T)^{2}}\right\} \\
& \times \sin ^{2}\left[N_{p} \epsilon_{s}(k ; T) T\right] \cos ^{2}\left(\phi_{\perp}^{k}+\phi_{g}\right),
\end{aligned}
$$

where $\epsilon_{s}(k ; T)=\frac{1}{2} \sqrt{\left(\omega_{\mathrm{av}}-k \omega\right)^{2}+\left|A_{\perp} f_{\perp}^{k}\right|^{2}}$ describes the $k$ th avoided crossing. The new parameter $\phi_{g}$ is introduced to denote a global phase added to all of the pulses while maintaining the phase of the initial $\mathrm{N}-V$ state and measurement. Recent work on spurious coherence dips has shown a dependence on global phase [25], and it is modeled here. Figure 5 shows a good fit of this analytic expression to numerical data.

At the dip $\epsilon_{s}\left(k ; T_{\text {dip }}^{k}\right)=\frac{1}{2}\left|A_{\perp} f_{\perp}^{k}\right|$, where $\left|A_{\perp} f_{\perp}^{k}\right|$ is the width of the quasienergy avoided crossing and defines the effective coupling strength of the nuclear spin to the $\mathrm{N}-V$ under DD control, as opposed to the bare coupling strength $A_{\perp}$. The term in curly brackets reaches unity at the dip position, so the depth of the dip is given by $\mathcal{L}_{s}\left(N_{p} T_{\text {dip }}^{k}\right)=1-2 \sin ^{2}\left(N_{p} \frac{1}{2}\left|A_{\perp} f_{\perp}^{k}\right| T_{\text {dip }}^{k}\right) \cos ^{2}\left(\phi_{\perp}^{k}+\phi_{g}\right)$, which reaches a maximum depth of $\mathcal{L}_{s}\left(N_{p}^{\max } T_{\text {dip }}^{k}\right)=$ $-\cos \left[2\left(\phi_{\perp}^{k}+\phi_{g}\right)\right]$ at $N_{p}=N_{p}^{\max }=\pi /\left(\left|A_{\perp} f_{\perp}^{k}\right| T_{\mathrm{dip}}^{k}\right)$. The depth of spurious dips is, therefore, limited by the phase of $f_{\perp}^{k}$. For $X Y 8, \phi_{\perp}^{k} \in\{ \pm \pi / 4, \pm 3 \pi / 4\}$, so the spurious dips will never drop below zero (for $\phi_{g}=0$ ). However, scanning $\phi_{g}$ gives control over the spurious-dip depths. In fact, the $k$ th spurious resonance can be turned off, to avoid ambiguities with other nuclear signals, by setting $\phi_{g}=$ $-\phi_{\perp}^{k} \pm \pi / 2$. Conversely, the contrast of a particular spurious dip can be maximized by choosing $\phi_{g}=-\phi_{\perp}^{k}$. Table I lists isotopes with fundamental signals that can
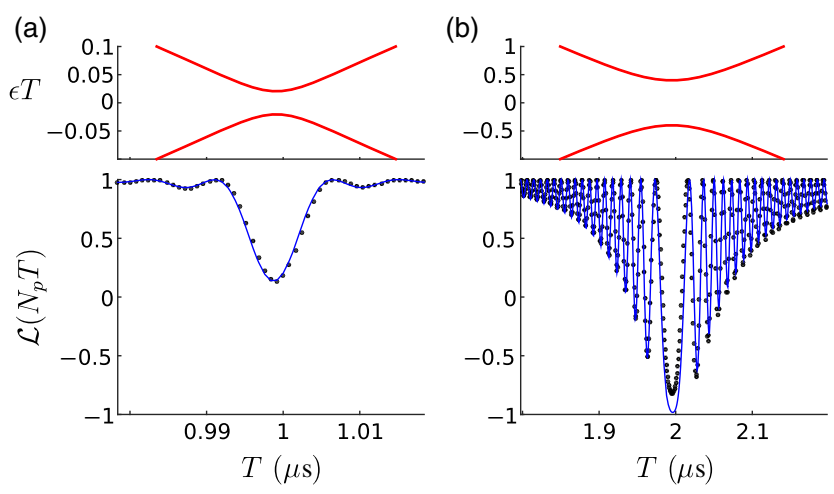

FIG. 5. Comparison of numerics (the black dots) to the analytic expressions (the blue lines) for (a) the $k=2$ spurious dip and (b) the $k=4$ expected dip under $X Y 8$ control. Above are the associated Floquet avoided crossings. Note that the scale on the expected avoided crossing is 10 times larger. For the numerical simulations here, in the Zeeman basis, $\omega_{z}=2 \pi \times 2 \mathrm{MHz}$ and $A_{x}=2 \pi \times 200 \mathrm{kHz}$. The pulse sequence has $N_{p}=N / 8=60$ repetitions. The finite pulses have the height $\Omega=2 \pi \times 20 \mathrm{MHz}$ and the duration $t_{p}=\pi / \Omega$.

be mimicked by the spurious harmonic of another isotope and lists the global phase required to suppress this spurious signal. These global phases are specific for the $X Y 8$ sequence, but similar relations can be derived for any other DD sequence.

For $N_{p}>N_{p}^{\max }$, the dip simply acquires additional sideband structures, which, with increasing $N_{p}$, progressively fill an envelope $\mathcal{L}_{s}^{\text {env }}(T)=1-2\left\{\left[\epsilon_{s}(k ; T)^{2}-\right.\right.$ $\left.\left.\left(\omega_{\mathrm{av}}-k \omega\right)^{2} / 4\right] /\left[\epsilon_{s}(k ; T)^{2}\right]\right\} \cos ^{2}\left(\phi_{\perp}^{k}+\phi_{g}\right)$ function, which is independent of $N_{p}$. We also find that this coherence envelope function has the width $W_{T}=2\left|A_{\perp} f_{\perp}^{k}\right| T_{\mathrm{dip}}^{k} / \omega_{\mathrm{av}}$.

A formula for the expected coherence dips can also be obtained by diagonalizing the Floquet Hamiltonian at $T_{\text {dip }}^{k}$ for $k$ such that $f_{z}^{k} \neq 0$ :

$\mathcal{L}\left(N_{p} T\right)=1-2\left(\frac{\epsilon(k ; T)^{2}-\left(\frac{\omega_{\mathrm{av}}-k \omega}{2}\right)^{2}}{\epsilon(k ; T)^{2}}\right) \sin ^{2}\left[N_{p} \epsilon(k ; T) T\right]$,

TABLE I. Isotopes susceptible to ambiguous characterization due to the presence of another isotope that mimics the signal at the listed harmonic. Applying the global phase, $\phi_{g}$, to all pulses in the $X Y 8$ sequence suppresses the unwanted spurious signal for unambiguous nuclear species classification. (For $X Y 8$, the $n \times$ harmonic of the fundamental signal is at $T_{\mathrm{dip}}^{k}=2 \pi k / \omega_{\mathrm{av}}$ for $k=4 / n$.)

\begin{tabular}{lccc}
\hline \hline Isotope & Mimic & Harmonic & $\phi_{g}$ \\
\hline${ }^{1} \mathrm{H}$ & ${ }^{13} \mathrm{C}$ & $4 \times$ & $-\pi / 4$ \\
${ }^{29} \mathrm{Si}$ & ${ }^{13} \mathrm{C}$ & $4 / 5 \times$ & $-\pi / 4$ \\
${ }^{31} \mathrm{P}$ & ${ }^{1} \mathrm{H}$ & $2 / 5 \times$ & $+\pi / 4$ \\
\hline \hline
\end{tabular}


where $\epsilon(k ; T)=\frac{1}{2} \sqrt{\left(\omega_{\mathrm{av}}-k \omega\right)^{2}+\left|A_{\perp} f_{z}^{k}\right|^{2}}$ describes the Floquet-eigenvalue avoided crossing, and there is no dependence on the phase of $f_{z}^{k}$ or a global phase. The parameters in this case have the same functional form but employ $f_{z}^{k}$ instead of $f_{\perp}^{k}$.

Since $f_{\perp}^{k} \ll f_{z}^{k}$, the width of the spurious dips is much less than those of expected dips implying an increase in resolution and a possible aid for improving sensing experiments. This result is described in Sec. IV.

\section{Comparisons with numerics}

To validate the new analytic expression for coherence dips, it is compared against a full numeric calculation. An example of the accurate fit is shown in Fig. 5. Both expected and spurious dips are shown to be modeled well by the analytic formula. The numerics are obtained by directly propagating the $\mathrm{N}-V$-nuclear-spin system in the Zeeman basis. Intervals of free propagation under $\hat{H}_{0}$ are concatenated with the propagation during the pulse under $\hat{H}_{0}+\hat{H}_{p}\left(t^{\prime}\right)$.

Notice that the $k=2$ spurious avoided crossing is approximately 20 times narrower than the expected $k=4$ avoided crossing. This narrowing equates to a 20 times reduction in dip width, as can be seen in the coherence traces. The spurious dips are much narrower than the expected dips. In the next section, we describe a protocol to exploit this feature to obtain increased resolution in sensing experiments.

\section{SPURIOUS DIPS AS A RESOURCE}

Recent theory proposals and experiments make use of tunable decoupling sequences to gain control over the effective coupling to nuclear spins [13,21-23]. These protocols involve the application of sequences made of composite pulses with adjustable interpulse spacing so that one can tune $\left|f_{z}^{k}\right|$ and open or close avoided crossings, i.e., augmenting or diminishing the effective coupling to nuclear spins - and thus the width of experimental signals. By choosing $\left|f_{z}^{k}\right|$ to be small, one can sharpen a selected coherence dip allowing for better resolution between isolated nuclear spins. The number of pulses that can be applied is constrained by the $T_{2}$ coherence time, so the minimal limit for $\left|f_{z}^{k}\right|$ is determined by the requirement that the dip still obtain a visible contrast after the maximum number of pulses.

One result of this work is to see that spurious dips are naturally much narrower than the normal dips because $\left|f_{\perp}^{k}\right| \ll\left|f_{z}^{k}\right|$. Hence, if spurious signals are observed in the spectra, one can increase the spectral resolution without complex pulse-sequence design. One requires only that $\left|f_{\perp}^{k}\right|$ is not so small that the dips cannot obtain appreciable contrast after the maximum allowed number of pulses is applied (this constraint is the same for tunable pulse sequences). From another point of view, the enhanced
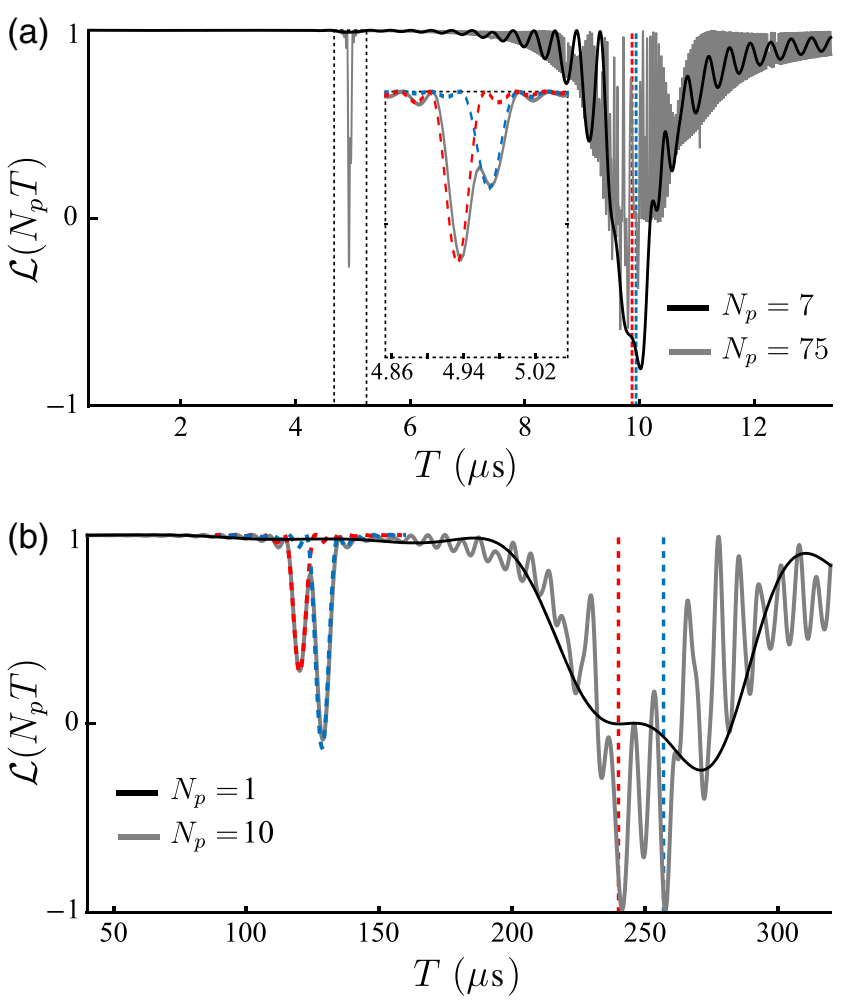

FIG. 6. (a) Numerical simulation of the $\mathrm{N}-V$ coherence coupled to two independent nuclear spins with $\omega_{\mathrm{av}}^{(1)}=2 \pi \times 402.6 \mathrm{kHz}$ and $\omega_{\mathrm{av}}^{(2)}=2 \pi \times 405.4 \mathrm{kHz}$ and hyperfine coupling strengths $A_{\perp}^{(1)}=2 \pi \times 21.6 \mathrm{kHz}$ and $A_{\perp}^{(2)}=2 \pi \times 31.0 \mathrm{kHz}$. (Solid black line) The coherence trace after $N_{p}=7$ repetitions of the $X Y 8$ sequence. (Solid gray line) The coherence trace after $N_{p}=75$ repetitions. The finite pulses have the height $\Omega=2 \pi \times 10 \mathrm{MHz}$ and the width $t_{p}=\pi / \Omega$. (Inset) A magnification of the spurious dips with the analytic expression, Eq. (15), for each dip plotted (the dashed line). (b) Numerical simulation of the $\mathrm{N}-V$ coherence coupled to two, more remote independent nuclear spins with $\omega_{\mathrm{av}}^{(1)}=2 \pi \times 16.67 \mathrm{kHz}$ and $\omega_{\mathrm{av}}^{(2)}=2 \pi \times 15.56 \mathrm{kHz}$ and hyperfine coupling strengths $A_{\perp}^{(1)}=2 \pi \times 1.63 \mathrm{kHz}$ and $A_{\perp}^{(2)}=2 \pi \times$ $2.14 \mathrm{kHz}$. (Solid black line) The coherence trace after $N_{p}=1$ repetitions of the $X Y 8$ sequence. (Solid gray line) The coherence trace after $N_{p}=10$ repetitions. The finite pulses have the height $\Omega=2 \pi \times 100 \mathrm{kHz}$ and the width $t_{p}=\pi / \Omega$. The analytic expression, Eq. (15), for the spurious dips is also plotted (the dashed line). In each case, the expected positions of the fundamental dip for each spin are denoted by the red and blue vertical dashed lines, but they are unresolved because the frequency separation is less than the hyperfine coupling strength. By increasing the number of pulses, the pairs of spins can be clearly resolved at the $k=2$ spurious dip. A global phase of $\phi_{g}=-\pi / 4$ is applied to the pulse sequence to enhance the contrast at the spurious dip.

spectral resolution by spurious dips is realized by making the effective coupling smaller than the frequency separation. Otherwise, the strong coupling invalidates the rotating-wave approximation to remove perturbation from unwanted spins [13,21]. 
This strategy has different benefits with respect to the use of more-complex sequences. On the one hand, the use of sequences containing composite pulses requires one to apply a number of pulses larger than the one used by a standard sequence, such as $X Y 8$. In this respect, and even under moderate pulse error conditions, the applicability of composite sequences can be challenging because the error accumulates, damaging the signal. On the other hand, the use of a robust sequence of composite pulses requires an accurate control of each pulse phase [13,21]—i.e., each pulse has to rotate the $\mathrm{N}-V$ state around a different axis on the $x-y$ plane-and this is another experimental requirement to be addressed. Note that, for a $X Y 8$ sequence, just two phases are needed. Furthermore, the analytic expression, Eq. (15), indicates that one can also gain control over the spurious dips, either by applying a global phase, $\phi_{g}$, to all pulses or by carefully designing the pulse sequence to selectively control $f_{\perp}^{k}$.

A simple protocol for increasing resolution is demonstrated in Fig. 6. Here, an $X Y 8$ sequence drives a N-V center coupled to two isolated nuclear spins $\left({ }^{13} \mathrm{C}\right.$ in diamond, for demonstration). In the first instance, the spins are approximately $0.6 \mathrm{~nm}$ from the $\mathrm{N}-V$, while, in the second instance, the spins are more weakly coupled at about $2 \mathrm{~nm}$ from the $\mathrm{N}-V$ center. In both cases, initial attempts to resolve the spin with the fundamental dip fail because the hyperfine coupling strengths are greater than the signal separation. However, by judiciously increasing the number of pulses and reducing the microwave pulse height (i.e., the Rabi frequency), one obtains sharp spurious features where the signals can be resolved. One can also add a global phase to the pulse sequence to enhance the contrast of the desired spurious dip.

\section{CONCLUSION}

We propose the use of the microwave pulse width, $t_{p}$ in dynamical-decoupling sequences as an additional experimental parameter in nanoscale NMR and MRI experiments. We demonstrate increased resolution in the detection of single nuclear spins by exploiting so-called spurious signals that arise due to finite pulse widths. We also propose methods for suppressing the spurious signals from nuclei when they mimic signals from other isotopes, thus removing ambiguity in nuclear-spin identification.

Floquet analysis is applied as the natural framework for studying quantum-sensing experiments under periodic dynamical-decoupling control. It reveals a landscape of avoided crossings in an underlying quantum spectrum that controls the position and the depth of characteristic $\mathrm{N}-V$ coherence dips. It is shown that adjusting $t_{p}$ from zero opens new avoided crossings that correspond to spurious signals in coherence traces. An analytic expression for spurious dips is given in Eq. (15), and it is shown to be in good agreement with numerics. Our study heralds an alternative generation of pulse-sequence design that exploits realistic pulse profiles alongside tuned pulse spacings and phases.

\section{ACKNOWLEDGMENTS}

The authors acknowledge their valuable discussions with Renbao Liu. J. E. L. acknowledges an EPSRC DTA studentship. J. C. acknowledges support to the Alexander von Humboldt Foundation. This work was supported by the ERC Synergy Grant BioQ, the EU projects DIADEMS and EQUAM, and the DFG Collaborative Research Center TRR 21.

\section{APPENDIX A: AVERAGE HAMILTONIAN STATES FOR SINGLE-SPIN SENSING}

To analyze the effect of finite pulses, we model an experiment sensing a single nuclear spin half, $\hat{\mathbf{I}}$. For nuclear spins far from the $\mathrm{N}-V$ center, we can assume the hyperfine coupling to be pure dephasing. In this case, the nuclear-spin Hamiltonian is conditioned on the state of the $\mathrm{N}-V$ center: $\hat{H}^{(0)}=\gamma_{n} B_{0} \hat{I}_{z_{\mathrm{N}-V}}$ and $\hat{H}^{(1)}=\gamma_{n} B_{0} \hat{I}_{z_{\mathrm{N}-V}}+$ $\mathbf{A} \cdot \hat{\mathbf{I}}$, where the superscripts 0 and 1 denote the $\mathrm{N}-V$-center state. Here, $\hat{\mathbf{z}}_{\mathrm{N}-V}$ is set by the $\mathrm{N}-V$ axis and $\hat{\mathbf{x}}_{\mathrm{N}-V}$ is set by the hyperfine field such that $\mathbf{A}=\left(A_{x}, 0, A_{z}\right)$. A magnetic field, $\mathbf{B}=B_{0} \hat{\mathbf{z}}_{\mathrm{N}-V}$, is applied parallel to the $\mathrm{N}-V$ axis and $\gamma_{n}$ is the nuclear gyromagnetic ratio. In the lab frame, the nuclear average Hamiltonian is given by $\hat{H}_{\mathrm{av}}=\gamma_{n} B_{0} \hat{I}_{z_{\mathrm{N}-\mathrm{V}}}+\frac{1}{2} \mathbf{A} \cdot \hat{\mathbf{I}}$, and the nuclear-interaction Hamiltonian is $\hat{V}=\frac{1}{2} \mathbf{A} \cdot \hat{\mathbf{I}}$.

In the average Hamiltonian basis, $\hat{H}_{\mathrm{av}}=\omega_{\mathrm{av}} \hat{I}_{z}$ and $\hat{V}=A_{\perp} \hat{I}_{x}+A_{\|} \hat{I}_{z}$, where $\hat{\mathbf{z}}=\cos \theta_{\mathrm{av}} \hat{\mathbf{z}}_{\mathrm{N}-V}+\sin \theta_{\mathrm{av}} \hat{\mathbf{x}}_{\mathrm{N}-V}$ and $\hat{\mathbf{x}}=\cos \theta_{\mathrm{av}} \hat{\mathbf{x}}_{\mathrm{N}-V}-\sin \theta_{\mathrm{av}} \hat{\mathbf{z}}_{\mathrm{N}-V}$. The average Hamiltonian frequency is $\omega_{\mathrm{av}}=\sqrt{\left(\gamma_{n} B_{z}+A_{z} / 2\right)^{2}+\left(A_{x} / 2\right)^{2}}$, and the hyperfine components in this basis are given by $A_{\perp}=\left(A_{x} \cos \theta_{\mathrm{av}}-A_{z} \sin \theta_{\mathrm{av}}\right) / 2$ and $A_{\|}=\left(A_{z} \cos \theta_{\mathrm{av}}+\right.$ $\left.A_{x} \sin \theta_{\mathrm{av}}\right) / 2$, where $\theta_{\mathrm{av}}=\arctan \left[A_{x} /\left(2 \gamma_{n} B_{0}+A_{z}\right)\right]$. Figure 3 shows the system fields in the two bases. For simplicity, we ignore the $A_{\|}$contribution to the interaction term as, for weak coupling, the effect of parallel fluctuations is small. In the Floquet Hamiltonian, this approximation can be understood, as it does not couple degenerate energy levels. Perturbative techniques could be used to study the effect of parallel coupling strengths.

Considering the application of a repeated $X Y 8$ dynamical-decoupling sequence, the $\mathrm{N}-V$-nuclear Hamiltonian can be written in the frame rotating under the effect of the pulse Hamiltonian as

$$
\hat{H}^{\prime}(t)=\hat{\rrbracket} \otimes \omega_{\mathrm{av}} \hat{I}_{z}+\sum_{i=x, y, z} f_{i}(t) \hat{\sigma}_{i} \otimes A_{\perp} \hat{I}_{x},
$$

where the modulation functions for $X Y 8$ are shown in Fig. 2. This Hamiltonian is shown in matrix form in 


$$
\hat{H}^{\prime}(t)=\left(\begin{array}{cccc}
\frac{\omega_{\mathrm{av}}}{2} & \frac{A_{\perp}}{2} f_{z}(t) & 0 & \frac{A_{\perp}}{2}\left[f_{x}(t)-i f_{y}(t)\right] \\
\frac{A_{\perp}}{2} f_{z}(t) & -\frac{\omega_{\mathrm{av}}}{2} & \frac{A_{\perp}}{2}\left[f_{x}(t)-i f_{y}(t)\right] & 0 \\
0 & \frac{A_{\perp}}{2}\left[f_{x}(t)+i f_{y}(t)\right] & \frac{\omega_{\mathrm{av}}}{2} & -\frac{A_{\perp}}{2} f_{z}(t) \\
\frac{A_{\perp}}{2}\left[f_{x}(t)+i f_{y}(t)\right] & 0 & -\frac{A_{\perp}}{2} f_{z}(t) & -\frac{\omega_{\mathrm{av}}}{2}
\end{array}\right)
$$

FIG. 7. Full N-V-nuclear-spin Hamiltonian in the average nuclear-spin basis. It is composed of the diagonal average Hamiltonian with periodic off-diagonal terms.
Fig. 7 so that it can be compared with a schematic representation of $\hat{H}_{F}$, which is shown in Fig. 4. The elements of the Floquet Hamiltonian, $\hat{H}_{F}$, are obtained from Eq. (5). In the weak-coupling regime, $\omega_{\mathrm{av}} \gg A_{\perp}, \hat{H}_{F}$ is approximately diagonal with only small off-diagonal perturbations.

The unperturbed eigenvalues are given by the diagonal entries $\epsilon_{ \pm l}= \pm \omega_{\mathrm{av}} / 2+l \omega$, which are doublets. These doublets remain degenerate, as there is no off-diagonal perturbation connecting them. More interesting are the degeneracies that occur when the DD frequency, $\omega$, is set to $\omega=\omega_{\mathrm{av}} / k$. At this point, $\omega_{\mathrm{av}} / 2+m \omega=-\omega_{\mathrm{av}} / 2+(m+$ $k) \omega$ for all $m$ 's. In Fig. 1(b), these unperturbed eigenvalues are plotted as $\epsilon_{ \pm l} T= \pm \omega_{\mathrm{av}} T / 2+l 2 \pi$.

If the off-diagonal perturbation connecting two diagonal entries is nonzero, the degeneracy will be lifted and an avoided crossing will appear. These perturbed eigenvalues are plotted in Figs. 1(c) and 1(d).

\section{APPENDIX B: COHERENCE FUNCTIONS}

\section{1. $t_{p}=0$ case: Expected coherence dips}

The Fourier amplitudes $f_{z}^{k}$ determine the positions of the expected coherence dips. The Fourier series for an $X Y 8$ sequence is shown in Fig. 2(c). Dips appear when $\omega=$ $\omega_{\text {av }} / k$ for $k=4,12,20, \ldots$. At these values of $\omega$, we must take the off-diagonal perturbations into account, and the Floquet Hamiltonian separates into an infinite set of $2 \times 2$ matrices which can be diagonalized.

As discussed in Sec. III B, the stroboscopic evolution in the original Hilbert space is obtained from the diagonalization of $\hat{H}_{F}$. At the expected coherence dips, the propagator is given by

$$
\hat{U}\left(N_{p} T\right)=e^{-i k N_{p} \pi}\left(\begin{array}{cccc}
u_{a} & u_{b} & 0 & 0 \\
-u_{b}^{*} & u_{a}^{*} & 0 & 0 \\
0 & 0 & u_{a} & -u_{b} \\
0 & 0 & u_{b}^{*} & u_{a}^{*}
\end{array}\right)
$$

where $u_{a}=\cos \left[N_{p} \epsilon(k ; T) T\right]-i \sin \left[N_{p} \epsilon(k ; T) T\right] \cos \theta_{F}(k ; T)$, $u_{b}=-i \sin \left[N_{p} \epsilon(k ; T) T\right] \sin \theta_{F}(k ; T) \quad$ and $\quad \epsilon(k ; T)=$ $\frac{1}{2} \sqrt{\left(\omega_{\mathrm{av}}-k \omega\right)^{2}+\left|A_{\perp} f_{z}^{k}\right|^{2}}$ and $\theta_{F}(k ; T)=\arctan \left[\left|A_{\perp} f_{z}^{k}\right| /\right.$ $\left.\left(\omega_{\mathrm{av}}-k \omega\right)\right]$.

The coherence of the $\mathrm{N}-V$ sensor near the dip at $T_{\text {dip }}^{k}=$ $2 k \pi / \omega_{\mathrm{av}}$ is thus modeled by

$$
\mathcal{L}\left(N_{p} T\right)=1-2 \sin ^{2}\left[N_{p} \epsilon(k ; T) T\right] \sin ^{2} \theta_{F}(k ; T) .
$$

In the main text, we use the identity $\sin ^{2} \theta_{F}(k ; T)=$ $\left[\epsilon(k ; T)^{2}-\left(\omega_{\text {av }}-k \omega\right)^{2} / 4\right] / \epsilon(k ; T)^{2}$ to emphasize the importance of the Floquet quasienergy spectra, $\epsilon(k ; T)$.

\section{2. $t_{p} \neq 0$ case: Spurious coherence dips}

For the $X Y 8$ sequence, there are spurious dips at $T_{\text {dip }}^{k}=$ $2 k \pi / \omega_{\mathrm{av}}$ for $k=1,2,3,5,6,7,9,10,11, \ldots$, as shown in Fig. 1. For these $k$ 's, the Floquet Hamiltonian will again have degeneracies in diagonal terms lifted by off-diagonal perturbations. The Floquet Hamiltonian separates into $2 \times 2$ matrices and can be diagonalized. The stroboscopic evolution is then given by

$$
\hat{U}_{s}\left(N_{p} T\right)=e^{-i k N_{p} \pi}\left(\begin{array}{cccc}
v_{a} & 0 & 0 & v_{b} \\
0 & v_{a}^{*} & -v_{b} & 0 \\
0 & v_{b}^{*} & v_{a} & 0 \\
-v_{b}^{*} & 0 & 0 & v_{a}^{*}
\end{array}\right),
$$

where $\quad v_{a}=\cos \left[N_{p} \epsilon(k ; T) T\right]-i \sin \left[N_{p} \epsilon_{s}(k ; T) T\right] \times$ $\cos \theta_{F s}(k ; T), \quad v_{b}=-i \sin \left[\left(N_{p} \epsilon_{s}(k ; T) T\right] \sin \theta_{F s}(k ; T) e^{-i \phi_{F}^{k}}\right.$ and $\epsilon_{s}(k ; T)=\frac{1}{2} \sqrt{\left(\omega_{\mathrm{av}}-k \omega\right)^{2}+\left|A_{\perp} f_{\perp}^{k}\right|^{2}}, \quad \theta_{F s}(k ; T)=$ $\arctan \left[\left|A_{\perp} f_{\perp}^{k}\right| /\left(\omega_{\mathrm{av}}-k \omega\right)\right]$ and $\phi_{F}^{k}=\phi_{\perp}^{k}$, where $f_{\perp}^{k}=$ $\left|f_{\perp}^{k}\right| e^{i \phi_{\perp}^{k}}$.

The spurious dip near $T_{\mathrm{dip}}^{k}=2 k \pi / \omega_{\mathrm{av}}$ in a N-V coherence trace is thus modeled by

$\mathcal{L}_{s}\left(N_{p} T\right)=1-2 \sin ^{2}\left[N_{p} \epsilon_{s}(k ; T) T\right] \sin ^{2} \theta_{F s}(k ; T) \cos ^{2} \phi_{F}^{k}$.

\section{APPENDIX C: CPMG SEQUENCES}

In this section, we briefly analyze the sensor response under the CPMG dynamical-decoupling sequence. A CPMG sequence has the same pulse positions as $X Y 8$ except that all pulses have the same $(x)$ phase. The pulse amplitudes are shown in Fig. 8(a) along with the modulation functions obtained from Eq. (11). The $f_{z}(t)$ modulation function is independent of the pulse phases and thus is the same as for $X Y 8$. The change of pulse phase is encoded into the perpendicular modulation functions. For CPMG, $f_{x}(t)=0$, while $f_{y}(t)$ is shown.

When detecting a single nuclear spin, as in the main text, one first studies the unperturbed Floquet spectrum to find that there are degeneracies at $T=2 \pi k / \omega_{\mathrm{av}}$. (We choose an eight-pulse CPMG unit 


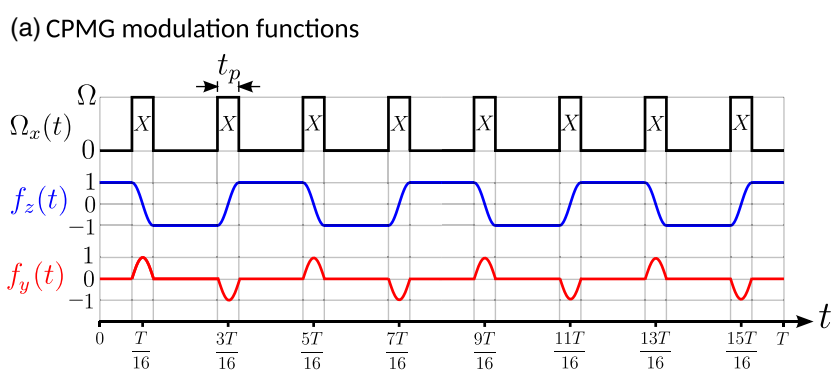

(b) CPMG coupling-matrix elements

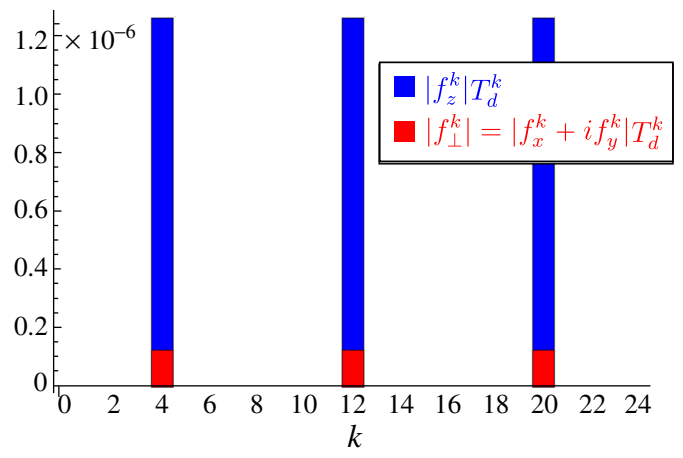

FIG. 8. (a) Shows the modulation functions for a CPMG sequence with pulses of finite duration $t_{p}$. (b) Matrix elements of the modulation function $\left\langle l+k\left|f_{i}\right| l\right\rangle$ in the dressed-state basis. For CPMG, the spurious couplings $f_{\perp}^{k}$ coincide with the expected couplings $f_{z}^{k}$. Thus, the presence of finite pulses perturbs the expected signal only slightly and does not produce new signals at spurious harmonics (as for $X Y 8$ ).

as one period to allow for a simple comparison with $X Y 8$.) Upon calculating the coupling-matrix elements in the Floquet Hamiltonian, one finds that the spurious couplings $A_{\perp} f_{\perp}^{k} / 2$ are nonzero for $k=4,12,20, \ldots$, coinciding with the nonzero expected couplings $A_{\perp} f_{z}^{k} / 2$; see Fig. 8(b). This correspondance is due to $f_{z}(t)$ and $f_{y}(t)$ having the same frequency, which immediately explains why spurious resonances are not seen in CPMG coherence traces. The spurious signal is buried inside the expected dip. In fact, the finite-pulse effect on CPMG traces is to perturb the expected coherence dip but not create new signals.

The coherence can again be modeled by $\mathcal{L}\left(N_{p} T\right) \propto$ $\operatorname{Tr}\left[\hat{S}_{x} \hat{U}\left(N_{p} T\right) \rho_{0} \hat{U}^{\dagger}\left(N_{p} T\right)\right]$, where the propagator at time $t=N_{p} T$ is determined by an effective Hamiltonian $\hat{U}\left(N_{p} T\right)=\exp \left(-i \hat{H}_{\mathrm{eff}} N_{p} T\right)$. At $T=2 \pi k / \omega_{\mathrm{av}}$,

$$
\hat{H}_{\mathrm{eff}}=\left(\begin{array}{cccc}
\frac{\omega_{\mathrm{av}}-k \omega}{2} & \frac{A_{\perp}}{2} f_{z}^{k} & 0 & \frac{A_{\perp}}{2} f_{\perp}^{k *} \\
\frac{A_{\perp}}{2} f_{z}^{k} & -\frac{\omega_{\mathrm{av}}-k \omega}{2} & \frac{A_{\perp}}{2} f_{\perp}^{-k *} & 0 \\
0 & \frac{A_{\perp}}{2} f_{\perp}^{-k} & \frac{\omega_{\mathrm{av}}-k \omega}{2} & -\frac{A_{\perp}}{2} f_{z}^{k} \\
\frac{A_{\perp}}{2} f_{\perp}^{k} & 0 & -\frac{A_{\perp}}{2} f_{z}^{k} & -\frac{\omega_{\mathrm{av}}-k \omega}{2}
\end{array}\right) .
$$

[1] R. Schirhagl, K. Chang, M. Loretz, and C. L. Degen, Nitrogen-vacancy centers in diamond: Nanoscale sensors for physics and biology, Annu. Rev. Phys. Chem. 65, 83 (2014).

[2] L. Rondin, J. P. Tetienne, T. Hingant, J. F. Roch, P. Maletinsky, and V. Jacques, Magnetometry with nitrogenvacancy defects in diamond, Rep. Prog. Phys. 77, 056503 (2014).

[3] Y. Wu, F. Jelezko, M. B. Plenio, and T. Weil, Diamond quantum devices in biology, Angew. Chem., Int. Ed. Engl. 55, 6586 (2016).

[4] N. Zhao, J. Honert, B. Schmid, M. Klas, J. Isoya, M. Markham, D. Twitchen, F. Jelezko, R. B. Liu, H. Fedder, and J. Wrachtrup, Sensing single remote nuclear spins, Nat. Nanotechnol. 7, 657 (2012).

[5] T. H. Taminiau, J. J. T. Wagenaar, T. Van der Sar, F. Jelezko, V. V. Dobrovitski, and R. Hanson, Detection and Control of Individual Nuclear Spins Using a Weakly Coupled Electron Spin, Phys. Rev. Lett. 109, 137602 (2012).

[6] S. Kolkowitz, Q. P. Unterreithmeier, S. D. Bennett, and M. D. Lukin, Sensing Distant Nuclear Spins with a Single Electron Spin, Phys. Rev. Lett. 109, 137601 (2012).

[7] A. O. Sushkov, I. Lovchinsky, N. Chisholm, R. L. Walsworth, H. Park, and M. D. Lukin, Magnetic Resonance Detection of Individual Proton Spins Using Quantum Reporters, Phys. Rev. Lett. 113, 197601 (2014).

[8] C. Müller, X. Kong, J.-M. Cai, K. Melentijevic, A. Stacey, M. Markham, D. Twitchen, J. Isoya, S. Pezzagna, J. Meijer, J. F. Du, M. B. Plenio, B. Naydenov, L. P. McGuiness, and F. Jelezko, Nuclear magnetic resonance spectroscopy with single spin sensitivity, Nat. Commun. 5, 4703 (2014).

[9] V. V. Mkhitaryan, F. Jelezko, and V. V. Dobrovitski, Highly selective detection of individual nuclear spins with rotary echo on an electron spin probe, Sci. Rep. 5, 15402 (2015).

[10] N. Zhao, J.-L. Hu, S.-W. Ho, J. T. K. Wan, and R. B. Liu, Atomic-scale magnetometry of distant nuclear spin clusters via nitrogen-vacancy spin in diamond, Nat. Nanotechnol. 6, 242 (2011).

[11] F. Shi, X. Kong, P. Wang, F. Kong, N. Zhao, R. B. Liu, and J. Du, Sensing and atomic-scale structure analysis of single nuclear-spin clusters in diamond, Nat. Phys. 10, 21 (2014).

[12] W. L. Ma and R. B. Liu, Angstrom-Resolution Magnetic Resonance Imaging of Single Molecules via Wave-Function Fingerprints of Nuclear Spins, Phys. Rev. Applied 6, 024019 (2016).

[13] Z.-Y. Wang, J. F. Haase, J. Casanova, and M. B. Plenio, Positioning nuclear spins in interacting clusters for quantum technologies and bioimaging, Phys. Rev. B 93, 174104 (2016).

[14] P. Cappellaro, L. Jiang, J. S. Hodges, and M. D. Lukin, Coherence and Control of Quantum Registers Based on Electronic Spin in a Nuclear Spin Bath, Phys. Rev. Lett. 102, 210502 (2009).

[15] G.-Q. Liu, H. C. Po, J. Du, R. B. Liu, and X.-Y. Pan, Noiseresilient quantum evolution steered by dynamical decoupling, Nat. Commun. 4, 2254 (2013).

[16] P. Neumann, R. Kolesov, B. Naydenov, J. Beck, F. Rempp, M. Steiner, V. Jacques, G. Balasubramanian, M. L. Markham, D. J. Twitchen, S. Pezzagna, J. Meijer, 
J. Twamley, F. Jelezko, and J. Wrachtrup, Quantum register based on coupled electron spins in a room-temperature solid, Nat. Phys. 6, 249 (2010).

[17] T. H. Taminiau, J. Cramer, T. Van der Sar, V. V. Dobrovitski, and R. Hanson, Universal control and error correction in multi-qubit spin registers in diamond, Nat. Nanotechnol. 9, 171 (2014).

[18] J. Casanova, Z.-Y. Wang, and M. B. Plenio, Noise-Resilient Quantum Computing with a Nitrogen-Vacancy Center and Nuclear Spins, Phys. Rev. Lett. 117, 130502 (2016).

[19] G. De Lange, Z. H. Wang, D. Riste, V. V. Dobrovitski, and R. Hanson, Universal dynamical decoupling of a single solid-state spin from a spin bath, Science 330, 60 (2010).

[20] J. Du, X. Rong, N. Zhao, Y. Wang, J. Yang, and R. B. Liu, Preserving electron spin coherence in solids by optimal dynamical decoupling, Nature (London) 461, 1265 (2009).

[21] J. Casanova, Z.-Y. Wang, J. F. Haase, and M. B. Plenio, Robust dynamical decoupling sequences for individualnuclear-spin addressing, Phys. Rev. A 92, 042304 (2015).

[22] N. Zhao, J. Wrachtrup, and R. B. Liu, Dynamical decoupling design for identifying weakly coupled nuclear spins in a bath, Phys. Rev. A 90, 032319 (2014).

[23] W. Ma, F. Shi, K. Xu, P. Wang, X. Xu, X. Rong, C. Ju, C.-K. Duan, N. Zhao, and J. Du, Resolving remote nuclear spins in a noisy bath by dynamical decoupling design, Phys. Rev. A 92, 033418 (2015).

[24] M. Loretz, J. M. Boss, T. Rosskopf, H. J. Mamin, D. Rugar, and C. L. Degen, Spurious Harmonic Response of Multipulse Quantum Sensing Sequences, Phys. Rev. X 5, 021009 (2015).

[25] J. F. Haase, Z.-Y. Wang, J. Casanova, and M. B. Plenio, Pulse-phase control for spectral disambiguation in quantum sensing protocols, Phys. Rev. A 94, 032322 (2016).

[26] L. Cywinski, R. M. Lutchyn, C. P. Nave, and S. Das Sarma, How to enhance dephasing time in superconducting qubits, Phys. Rev. B 77, 174509 (2008).

[27] J. E. Lang, R. B. Liu, and T. S. Monteiro, DynamicalDecoupling Based Quantum Sensing: Floquet Spectroscopy, Phys. Rev. X 5, 041016 (2015).

[28] T. Guillion, D. B. Baker, and M. S. Conradi, New, compensated Carr-Purcell sequences, J. Magn. Reson. 89, 479 (1990).

[29] H. Y. Carr and E. M. Purcell, Effects of diffusion on free precession in nuclear magnetic resonance experiments, Phys. Rev. 94, 630 (1954).

[30] S. Meiboom and D. Gill, Modified spin-echo method for measuring nuclear relaxation times, Rev. Sci. Instrum. 29, 688 (1958). 